\title{
ANXIETY AND DEPRESSION IN PATIENTS WITH TINNITUS
}

\section{Sikandar Ali Khan, Maqbool Raza, Shahid Farooq*, Muhammad Tariq**, Muzafar Chandio, Atif Rafique}

\author{
Combined Military Hospital Multan/National University of Medical Sciences (NUMS) Pakistan, *Combined Military Hospital Peshawar/National University of
} Medical Sciences (NUMS) Pakistan, ${ }^{* *}$ Combined Military Hospital Jhelum/National University of Medical Sciences (NUMS) Pakistan

\section{ABSTRACT}

Objective: To determine the presence and severity of depression and anxiety in patients with tinnitus.

Study Design: Cross sectional study.

Place and Duration of Study: This study was conducted at department of Psychiatry, Combined Military Hospital Multan in collaboration with ENT department, Combined Military Hospital Multan, from Sep 2019 to Mar 2020.

Methodology: All diagnosed cases of tinnitus in ENT out-patient department Tinnitus Handicap inventory (THI) was applied to assess the severity of tinnitus. Diagnosis of depression and Anxiety was done based on diagnostic criteria of ICD10 of WHO and symptom severity was assessed using Beck Depressive Inventory (BDI) for depression and Beck Anxiety Inventory (BAI) for Anxiety.

Results: A total of 88 participants were included in this study. Fifty-three (60.2\%) were male, $35(39.7 \%)$ were females. Twentythree $(20.2 \%)$ ranged between 18-40 years of age, $65(57.2 \%)$ participants ranged between 41-60 years of age. Seven $(12.5 \%)$ participants scored less than 38 on tinnitus handicap inventory showing mild severity of tinnitus, $3(5.3 \%)$ individuals scored moderate while $19(33.9 \%)$ had severe level of tinnitus. Thirty-three (37\%) participants had depression $55(62 \%)$ out of total 88 participants had anxiety.

Conclusion: Studies show presence of anxiety and depression in patients with tinnitus so it seems prudent to screen all patients of tinnitus for psychiatric co morbidity. A multidisciplinary approach is required for the treatment of patients with tinnitus so that physical as well as psychiatric/psychological management of these patients is provided well in time.

Keywords: Anxiety, Depression, Tinnitus.

How to Cite This Article: Khan SA, Raza M, Farooq S, Tariq M, Chandio M, Rafique A. Anxiety and Depression in Patients with Tinnitus. Pak Armed Forces Med J 2021; 71 (Suppl-3): S517-520 Doi: https://doi.org/10.51253/pafmj.v1i1.7919

This is an Open Access article distributed under the terms of the Creative Commons Attribution License (https://creativecommons.org/licenses/by-nc/4.0/), which permits unrestricted use, distribution, and reproduction in any medium, provided the original work is properly cited.

\section{INTRODUCTION}

Tinnitus is an auditory sensation felt subjectively by the individual unilaterally or bilaterally in ears or head in the absence of external source. ${ }^{1}$ The auditory sensation can be a hiss, buzz, whistle, rain, water fall or any other sound or it can be an ill-defined sound which can be continuous or episodic. ${ }^{2}$ Tinnitus is a symptom of unknown cause. ${ }^{3}$ Which occurs in approximately $10-20 \%$ of people, ${ }^{4}$ most individual experiencing tinnitus have neutral reaction but some develop distress or negative emotions. ${ }^{5}$ Tinnitus can lead to difficulty in sleeping, concentrating in addition to compromised quality of life. ${ }^{6}$ Tinnitus is usually associated with hearing loss but studies has also shown that tinnitus is correlated with anxiety and depression. ${ }^{7}$ Studies have shown that it is difficult to establish causal effect of tinnitus with anxiety and depression but on the other hand there are studies that show association of tinnitus with depression and anxiety. Different studies show wide range in rate of depression and anxiety among patients with tinnitus. Rate of depression in different studies range from $9-90 \%$ of patients of tinnitus

Correspondence: Dr Sikandar Ali Khan, Assistant Professor of Psychiatrist, Combined Military Hospital, Multan Pakistan while rate of anxiety ranged from $10-95 \%$ in patients with tinnitus. ${ }^{8}$

To assess the impact of tinnitus clinicians rely on self-report questionnaires that measure and identify severity of tinnitus along with difficulties/negative consequences/limitations caused by tinnitus. There is no consensus upon how to assess these difficulties. Most commonly used instrument to test tinnitus is Tinnitus handicap inventory (THI) as there is no other objective test available for tinnitus, with help of tinnitus handicap inventory it is easier to determine the subjective tinnitus symptoms. ${ }^{10}$ Tinnitus handicap inventory probes into problems with function, emotions and catastrophizing. ${ }^{11}$ Tinnitus functional index is more recently developed questionnaire used that covers intrusiveness, sense of control, cognitions, sleep, auditory, relaxation, quality of life and emotional impact of tinnitus to measure the severity of tinnitus. ${ }^{12}$

Tinnitus is a prevalent complaint, and people with bothersome tinnitus can report any number of associated problems like depression and anxiety. Yet, no local studies or data is available on this topic. Our primary objective is to determine psychological impact of tinnitus in our own setup so as to provide base line 
understanding for clinicians and pave the way for future research on the psychological impact of tinnitus.

\section{METHODOLOGY}

After calculating sample size as per Kelsey et al,13 total of 88 individuals with tinnitus were included by using convenience non probability sampling technique after the approval of ethical committee. This cross-sectional study was conducted at department of Psychiatry, Combined Military Hospital Gujranwala in liaison with department of ENT, from September 2019 to March 2020. After written consent all were explained purpose of this study and confidentiality was ensured. Subjects that fulfilled following criteria were included: the study group; individuals of 18-50 years oldwith tinnitus. Individuals with past psychiatric illness were not included. Demographic details were documented. To assess the severity level of tinnitus "Tinnitus Handicap Inventory Severity Scale" (THI) was applied. ${ }^{4}$ THI is self-reporting questionnaire that has 25 questions, for each question there are three responses (Yes/ Sometimes/No), each of response has score of 1 . For scoring total numbers of "Yes" responses is multiplied by 4 , total numbers of "Sometimes" is multiplied by 2 while numbers obtained from the response of "No" is multiplied by 0 . After multiplication the sums are added to get the total score. Scores falling between 0-16 means slight severity of tinnitus, scores of 18-36 show mild severity, 38-56 moderate severity, 58-76 de-picts severe level of tinnitus, 78-100 scores are labelled as catastrophic level of severity. Slight severity means that tinnitus can very easily be masked with no interference with sleep or daily activities. Mild tinnitus means that it can be easily masked by environmental sounds and forgotten with activities, it may occasionally interfere with sleep but not daily activities. In moderate tinnitus it is noticed even in the presence of background or environmental noise although daily activity may still be performed. In severe level of tinnitus sounds are always heard, rarely if ever masked this leads to disturbed sleep and can interfere with daily activities, quiet activities are adversely affected. In catastrophic level of severity sounds are always heard sleep is disturbed with difficulty in any activity. Detailed assessment and diagnosis of depression and anxiety was done based on diagnostic criteria of ICD10 of WHO and symptom severity was assessed using Beck Depressive Inventory (BDI) for depression and Beck Anxiety Inventory (BAI) for anxiety. Beck Depressive Inventory consists of 21 questions each with four possible answers that are assigned a score ranging from 0-3. Higher scores indicate more severe symptoms. Total score is obtained by adding all scores of 21 items, total score ranges from 0-63. The cut off score for presence of symptoms is. ${ }^{9}$ Beck Depressive Inventory is for detecting symptoms not for diagnosing depression. Scores of 0-9 indicate minimal depression, 10-18 mild depression, 19-29 moderate depression and scores of 30-63 indicate severe depressive symptoms. Beck Anxiety Inventory (BAI) consists of 21 items, scored on Likert-type scale from $0-3 ; 0$ as not at all, 1 as mild, 2 as moderate, 3 as severe. Total score of $0-7$ reflects minimal anxiety, 8-15 mild anxiety, 16-25 moderate anxiety and 26-63 reflects severe anxiety. Statistical data is presented in frequencies and percentages.

\section{RESULTS}

A total of 88 participants were included in this study. Fifty three $(60.2 \%)$ were male, $35(39.7 \%)$ were females. Twenty three (20.2\%) ranged between 18-40 years of age out of which $16(71 \%)$ were males and $7(29 \%)$ were females, $65(57.2 \%)$ participants ranged between 41-60 years of age out of which 37 (57\%) were male and $28(39.7 \%)$ were females. Seventy nine $(89.7 \%)$ of participants were married while $9(10.3 \%)$ were unmarried. None of the participants of study was illiterate, 41 (47\%) secondary educated, 26 (29.3\%) had higher education, $13(15 \%)$ primary educated while only $8(8.7 \%)$ were graduate. Fifty nine $(67 \%)$ were employed and $29(32.9 \%)$ were unemployed. Seven $(12.5 \%)$ participants scored $<38$ on tinnitus handicap inventory showing mild severity of tinnitus, $3(5.3 \%)$ individuals scored moderate while 19 (33.9\%) had severe level of tinnitus. Thirty three $(37 \%)$ participants had depression out of which 17 (53.7\%) were males that had depression while $16(46.3 \%)$ females had depression. Amongst 17 males that had depression it was seen that $4(27 \%)$ had mild level of depression, 12 $(71 \%)$ had moderate while $1(2 \%)$ had severe level of depression (table-II). Amongst 16 females that had depression $6(38.7 \%)$ suffered from mild level of depression, $9(60.3 \%)$ had moderate while $1(1 \%)$ female suffered from severe level of depression (table-II). Fifty five $(62 \%)$ out of total 88 participants had anxiety out of which $40(72.7 \%)$ were females while $15(27.3 \%)$ were males that had anxiety (Table-I). amongst those 40 females that had anxiety $9(21 \%)$ had mild anxiety, $27(68 \%)$ had moderate while $4(11 \%)$ female had severe level of anxiety. Amongst those 15 males that had anxiety $2(13 \%)$ had mild level of anxiety, $12(82 \%)$ had moderate while $1(5 \%)$ male suffered severe level of anxiety (table-II). 


\section{DISCUSSION}

In this study, 23 (20.2\%) participants aged between $18-40$ years of age with mean age $31.2 \pm 14$ out of which $16(71 \%)$ were males and seven $(29 \%)$ were females, $65(57.2 \%)$ of participants age ranged between 41-60 years of age with mean age $53 \pm 16.2$ out of which 37 (57\%) were males and 28 (43\%) were females. In study by McCormicket al ratio of female participants was higher (54\%) as compared to male participants $(46 \%)^{8}$ similar to studies done by Traviset al in which female participants were $57 \%$ while male participants were $45 \% .{ }^{14,15}$ In our study, majority of participants were males $(60.2 \%)$ as compared to female participants $39.7 \%$. A total of $89.7 \%$ of participants were married while only $10.3 \%$ were unmarried. None of the participant was illiterate, $91 \%$ were undergraduate while only $8 \%$ of participants were graduate. Total of $67 \%$ of participants were employed while $32.9 \%$ were unemployed.

Pilgrim et al,In his study has noted that tinnitus is more frequently present in females similar to the observation done by Strumilla, ${ }^{16}$ on the other hand results of study by McCormicket al show that tinnitus is more frequently present in males as compared to females. ${ }^{15}$ In our study, we have focused on the level of severity of tinnitus present in our participants which has revealed that $29(51.7 \%)$ of participants had tinnitus out of which 7 (12.5\%) had mild level of tinnitus, 3 (5.3\%) had moderate while 19 (33.9\%) of participants had severe level of tinnitus. Salviati in his study has noted that psychiatric comorbidity in patients of tinnitus is higher in females $(56.8 \%)$ as compared to males $(42.3 \%){ }^{2}$ Strumilla suggests that severe level of tinnitus is associated with depression and anxiety which is higher in females. ${ }^{16}$ Similarly, McCormicket al has shown association of depression and anxiety with tinnitus ${ }^{8}$ but on the contrary Al-Rawashdeh notes that tinnitus is predictor of depression not anxiety. ${ }^{17,18}$ In our study total of 33 (37\%) of participants had depression out of which 17 (53.7\%) were males and $16(46.3 \%)$ were females that had depression. When level of severity was assessed 4 $(27 \%)$ males out of 17 had mild level of depression, 12 (71\%)males had moderate while 1 (2\%) male out of 17 had severe level of depression. On the other hand 6 $(38.7 \%)$ out of 16 female participants with depression had mild, $9(60.3 \%)$ females had moderate while $1(1 \%)$ out 16 females with depression had severe level of depression.

In our study, total of $55(62 \%)$ of participants exhibited anxiety out of which $15(27.3 \%)$ were males and $40(72.7 \%)$ were females. When level of severity of anxiety was assessed it was noted that $2(15 \%)$ out of fifteen males had mild anxiety, 12 (82\%) out of fifteen males had moderate while $1(5 \%)$ out of fifteen males had severe level of anxiety. On the other hand $9(21 \%)$ out 40 females exhibited mild anxiety, 27 (68\%) out of 40 females had moderate while $4(11 \%)$ out of 40 females exhibited severe level of anxiety.

Results of this study clearly show that anxiety is more frequent than depression in patients having tinnitus. Depression is noted more in males while anxiety is seen higher in females. These results differ from the results of Adogaet al ${ }^{4}$ who has noted that males had higher level of anxiety $(26.2 \%)$ as compared to females $(20 \%)$. Trevis et al ${ }^{14}$ in his study has shown that $95 \%$ of his participants exhibited anxiety while only $28 \%$ of his participants had depression. Bhatt, ${ }^{18}$ in his study has shown that $26.1 \%$ of his participants exhibited anxiety while $25.6 \%$ had depression with tinnitus as compared to the results of this study in which $62 \%$ of the participants have anxiety and 37\% of participants have depression as comorbidity with tinnitus.

Studies show wide range in rate of depression and anxiety among patients with tinnitus. Rate of depression in different studies range from $9-90 \%$ of patients of tinnitus while rate of anxiety ranged from 10$95 \%$ in patients with tinnitus. ${ }^{8}$

\section{CONCLUSION}

The presence of mental disorders is more common with tinnitus specifically depressive and anxiety disorders. The psychological/psychiatricinterventioninpatients of tinnitus and choosing appropriate management may help the patients to cope with the stress caused by tinnitus and assist them to adjust in their normal daily activities.

\section{Conflict of Interest: None.}

\section{Authors' Contribution}

SAK: Data collection, writing MR: Data collection, statistical analysis SF: Writing MT: Data collection MC: Writing AR: Statistical analysis, proof reading

\section{REFERENCES}

1. Moon KR, Park S, Jung Y, Lee A, Lee JH. Effects of anxiety sensitivity and hearing loss on tinnitus symptom severity. Psych Investigat 2018; 15(1): 34-38

2. Salviati M, Bersani FS, Terlizzi S, Melcore C, Panico R, Romano GF, et al. Tinnitus: clinical experience of the psychosomatic connection. Neurop DiseasTreat 2014;10(1):267-270.

3. Gibrin PC, Ciquinato DS, Gonçalves IC, Marchiori VD, Marchiori LL. Tinnitus and its relationship with anxiety and depression in the elderly: a systematic review. Revist Cefac 2019; 21(4): 1-5.

4. Adoga AA, Adoga AS, Obindo JT. Tinnitus and the prevalence of co-morbid psychological stress. Niger J Med 2008; 17(1): 95-97. 


\section{Anxiety and Depression}

5. Asnis GM, Majeed K, Henderson MA, Sylvester C, Thomas M, La Garza RD. An examination of the relationship between insomnia and tinnitus: a review and recommendations. Clinical Medicine Insights: Psych 2018; 9(1): 1179557318781078.

6. Cima RF, Mazurek B, Haider H, Kikidis D, Lapira A, Noreña A, et al. A multidisciplinary European guideline for tinnitus: diagnostics, assessment, and treatment. Hno 2019; 67(1): 10-42.

7. Hall DA, Fackrell K, Li AB, Thavayogan R, Smith S, Kennedy V, et al. A narrative synthesis of research evidence for tinnitusrelated complaints as reported by patients and their significant others. Health Quality Life Outcom 2018; 16(1): 61-65.

8. McCormack A, Edmondson-Jones M, Fortnum H, Dawes PD, Middleton $\mathrm{H}$, Munro KJ, et al. Investigating the association between tinnitus severity and symptoms of depression and anxiety, while controlling for neuroticism, in a large middle-aged UK population. Inter J Audiol 2015; 54(9): 599-604.

9. Watts EJ, Fackrell K, Smith S, Sheldrake J, Haider H, Hoare DJ. Why is tinnitus a problem? A qualitative analysis of problems reported by tinnitus patients. Trends Hearing 2018; 22(2): 2331216518812250.

10. Ziai K, Moshtaghi O, Mahboubi H, Djalilian HR. Tinnitus patients suffering from anxiety and depression: a review. Inter Tinnit J 2017; 21(1): 68-73.

11. Malakouti SK, Mahmoudian MN, Alifattahi N, Salehi M. Comorbidity of chronic tinnitus and mental disorders. Inter Tinnit J 2011; 16(2): 118-22.

12. Henry JA, Griest S, Thielman E, McMillan G, Kaelin C. Tinnitus functional index: development, validation, outcomes research, and clinical application. Hearing Res 2016; 334(2): 58-64.

13. Kelsey. Methods in observational epidemiology. $2^{\text {ndedition, }}$ Available at: https://www.amazon.com/Methods-Observational-Epidemiology-Jennifer-Kelsey/dp/0195083776
14. Trevis KJ, McLachlan NM, Wilson SJ. A systematic review and meta-analysis of psychological functioning in chronic tinnitus. Clinical Psychol Rev 2018; 60(1): 62-86.

15. Ziai K, Moshtaghi O, Mahboubi H, Djalilian HR. Tinnitus patients suffering from anxiety and depression: a review. Inter Tinnit J 2017; 21(1): 68-73.

16. Strumila R, Lengvenyte A, Vainutiene V, Lesinskas E. Influence of personality traits on tinnitus severity perception, anxiety and depressive symptoms. Psych Q 2017; 88(4): 865-877.

17. Al-Rawashdeh BM, Saleh MYN, Mustafa RB, Alkhouja MF, Elkhatib AH, Sughaireen $\mathrm{H}$, et al. Prevalence of depression and anxiety among otolaryngology outpatients at Jordan University Hospital. Perspect Psych Care 2018; 3(1): 1-13.

18. Bhatt JM, Bhattacharyya N, Lin HW. Relationships between tinnitus and the prevalence of anxiety and depression. Laryng 2017; 127(2): 466-469.

19. Meyer M, Neff P, Grest A, Hemsley C, Weidt S, Kleinjung T. EEG oscillatory power dissociates between distress-and depressionrelated psychopathology in subjective tinnitus. Brain Res 2017; 1663(2): 194-204.

20. Pattyn T, Van Den Eede F, Vanneste S, Cassiers L, Veltman DJ, Van De Heyning $P$, et al. Tinnitus and anxiety disorders: a review. Hearing Res 2016; 333(2): 255-265.

21. Pinto PC, Marcelos CM, Mezzasalma MA, Osterne FJ, de Lima MD, Nardi AE. Tinnitus and its association with psychiatric disorders: systematic review. J Laryngol Otol 2014; 128(8): 660664.

22. Landgrebe M, Langguth B. Tinnitus and anxiety. In Text Book Tinnit 2011; 1(1): 499-503.

23. Langguth B. A review of tinnitus symptoms beyond 'ringing in the ears': a call to action. Current Med Res Opinion 2011; 27(8): $1635-1643$ 